\section{Handbook of Mass Spectra of Environmental Contaminants, Second Edition}

Ronald L. Hites

Lewis Publishing Company

ISBN \#0-87371-534-9, \$89.95, Outside U.S.: \$108.00

CRC Press, Inc., 2000 Corporate Blvd., NW

Boca Raton, FL 33431

Book review by Michael L. Gross

Department of Chemistry

University of Nebraska

Lincoln, NE 68588

This book is a compilation of electron ionization mass spectra of 533 common environmental pollutants. The compounds and their spectra were taken from U.S. Environmental Protection Agency databases and the Merck Index.

The spectra are generously presented-one spectrum per page. Included with the spectrum are the compound name, Merck number, CAS number, formula, exact molecular mass, and a list of the four most abundant ions. The structure is given on the spectrum, and a few of the product ions are labeled in terms of their origin.

In an effort to remove errors, the spectra were reviewed by two experienced mass spectrometrists before publication.

A quick check of compounds familiar to this reviewer revealed that spectra of two isomers of tetra- chlorodibenzodioxin, of octachlorodibenzodioxin, and of octachlorodibenzofuran were given. No spectra of any other congeners or isomers of these compound classes were provided. The spectra agreed well with ones we have obtained at the University of Nebraska.

Another class of compounds of interest to us are the polycyclicaromatic hydrocarbons. The spectra of the highly carcinogeruic benzola]pyrene and 7,12-dimethylbenz[a]anthracene are part of the collection.

The spectra of many polar compounds are given: such as those of 2,4-D, 2,4,5-T, pentachlorophenol, etc. Because these types of materials are often derivatized bcforc analysis by GC/MS, a useful addition for future volumes would be the spectra of common derivatives of polar herbicides and pesticides.

The order of presentation of spectra is random, and one wonders why an arrangement in terms of increasing molecular weight was not chosen. The inconvenience, however, is offset by the indices at the back of the book. Here, the compounds are arranged in four indices: alphabetical in terms of common names, numerical according to the CAS Registry Number, numerical according to increasing exact molecular mass, and numerical according to increasing base peak in the EI spectra.

I have found the book a useful and convenient resource for rapidly locating a mass spectrum and for providing unknowns for students who are learning to interpret mass spectra. I was surprised, however, that the collection is apparently not available on diskette for computer use. 